\title{
Electrical Vehicle Speed Control by AI Technique
}

\author{
Mahesh Ramesh Birajdar \\ Student \\ Electrical Engineering dept. \\ Ashokrao Mane Group of Institutions, \\ Vathar \\ maheshbirajdar1998@gmail.com \\ Pratik Sudhakar Jangam \\ Student \\ Electrical Engineering dept. \\ Ashokrao Mane Group of Institutions, \\ Vathar \\ pratikj917010@gmail.com
}

\author{
Tabasum Bashir Patait \\ Student \\ Electrical Engineering dept. \\ Ashokrao Mane Group of Institutions, \\ Vathar \\ tabasumpatait@gmail.com \\ Swpnil Sudhakar Bhosle \\ Student \\ Electrical Engineering dept. \\ Ashokrao Mane Group of Institutions, \\ Vathar \\ bswapni1699@gmail.com
}

\author{
Alisha Rafik Sayyad \\ Student \\ Electrical Engineering dept. \\ Ashokrao Mane Group of Institutions, \\ Vathar \\ alishasayyad148@gmail.com \\ Ashish Anna Malgave \\ Head \\ Electrical Engineering dept. \\ Ashokrao Mane Group of Institutions, \\ Vathar \\ ashish_malgave@rediffmail.com
}

\begin{abstract}
This research paper in regards to the programmed vehicle driving framework without driver. This part presents a speed control of electric vehicle (EV) to improve the comportment and solidness under different street essential state . The control circuit utilizing shrewd fuzzy PI regulator is proposed. Boundaries which manage the working of PI regulator are progressively changed with the help of fuzzy canny control. This work manages the issue of way following for an electrical vehicle which has four electromechanical wheels framework. A regulator dependent on fuzzy rationale send the speed and the guiding guidelines to the robot to guarantee that the robot follows the direction, during the plan of this regulator the exertion was focused on the effortlessness and proficiency. The handling and reaction time are fundamental components in the process control, which is the thing that roused the decision of the construction and the boundaries of the various pieces of the fuzzy regulator
\end{abstract}

\section{Keywords-electric vehicle, fuzzy logic controller}

\section{INTRODUCTION}

This part presents a speed control of electric vehicle (EV) to improve the comportment and solidness under different street essential state. The control circuit utilizing shrewd fuzzy PI regulator is proposed. Boundaries which manage the working of PI regulator are progressively changed with the help of fuzzy wise control. All things considered, electric vehicle (EV) including, full cell and crossover vehicle have been grown quickly as an answer for energy and natural issue.. A self-driving vehicle is a vehicle that can run selfgoverning utilizing a control. There are two frameworks that are controlled in this exploration. The primary speed control capacities to manage the speed and development of oneself driving vehicle model by changing the PWM esteem on the DC engine and second, the controlling control utilizes the

Ackerman directing framework with a servo engine as an actuator. Both are masterminded utilizing fuzzy rationale control techniques that adjust from the propensities in driving a vehicle. the objective self-driving vehicle model can stroll to follow the track, keep up the robot vehicle in the path, can change the speed when turning and can stop when there are deterrents. These wheel engines can be controlled autonomously and have so brisk and exact reaction to the order that the vehicle skeleton control or movement control turns out to be more steady and strong, contrasted with in a roundabout way determined EVs.

Exploration has shown that EV control techniques, for example, PI control can perform ideally preposterous scope of activity conditions and aggravations and it is successful with consistent vehicle force, Moreover these non-direct vehicle force are not fixed and change haphazardly. There are additionally conditions where the turning point that is passed shifts the speed when turning likewise should be changed so as not to slip. In light of these conditions, the fuzzy rationale control technique was picked to have the option to deliver activities that relate to these conditions. The control of oneself driving vehicle model that is made is as per the plan of oneself driving vehicle model that can follow the direction however oneself driving vehicle model a few times is still out of the track on the grounds that oneself driving vehicle model doesn't have the correct bearing when turning. Self-driving vehicle models can back off when going to change the directing point and can stop when there are obstructions or traffic signals.

\section{ROBOTCAR MODULING}

The displaying is a significant advance before the utilization of the control, the more the demonstrating considered all the power applied on the framework the more the outcome are effective. The primary unique modulation of the vehicle that we utilized are create by yet he doesn't take on thought every one of the powers, this model improved by adding some power to thought, we utilized the powerful model improved in this work.

Our work depends on the control investigation of a selfsufficient electrical vehicle that presents four wheels driven by DC engine, the slowing down is finished by electromagnetic brakes when the shortfall of flow it additionally has double front directing framework and back

\section{A. Path Providing}

In these new days, many line following robots have been planned and used. This line following robots ordinarily comprises of either four wheels, two wheels or single wheel. The line following robot was intended to follow a line that might be an actual spot on the floor. The sensor was mounted with the robot to speak with microcontroller in 
detecting the line that had drawn by line tracer on a white surface or the other way around. The venture is developed utilizing an Arduino Board (UNO) as a microcontroller, two dc gear engines, 12V DC supply, ultrasonic sensor, and robot outline. The square outline of the framework is appeared in Figure 1. The reason for the paper is to give easier robots equipment engineering.

At startup, the four engines are turned on all the while, making the robot push ahead. Then, the ultrasonic sensor conveys beats from the front of the robot at customary stretches. On the off chance that an impediment is available, the sound heartbeats are reflected and are identified by the sensor. The impression of heartbeats relies on the actual condition of the obstruction: in the event that it is unpredictable fit as a fiddle, the beats reflected will be less; assuming it is uniform, the majority of the communicated heartbeats will be reflected. The reflection likewise relies on the course of the deterrent. On the off chance that it is marginally shifted, or put in corresponding with the sensor, the greater part of the sound waves will pass un reflected.

At the point when an obstruction is distinguished before the robot, at that point the side yields from the IR sensors are noticed. In the event that a hindrance is distinguished on the correct side, the left side tires of the robot are debilitated, making it turn towards the left, and the other way around. Assuming an impediment isn't recognized, the calculation is rehashed.

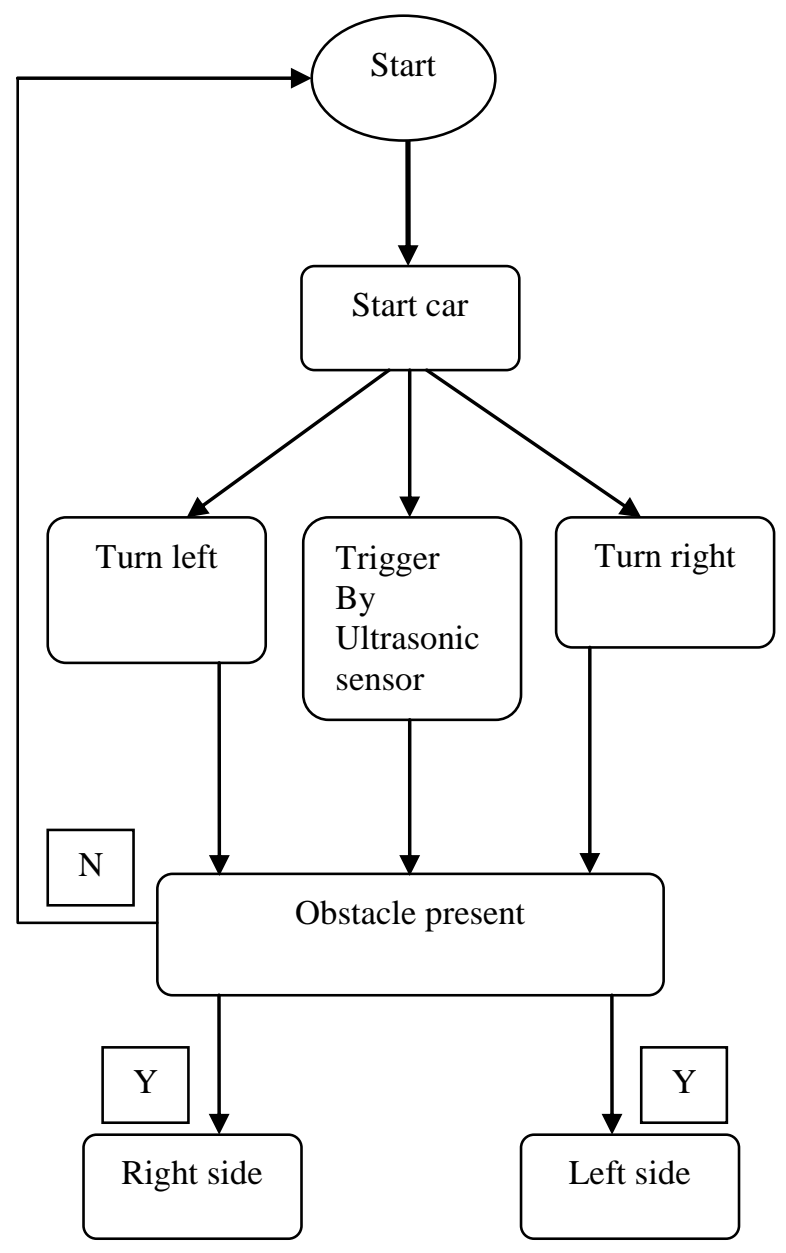

Fig. 1.
A ultrasonic sensor is a gadget that can quantify the distance to an article by utilizing sound waves. Sound goes through air at around $344 \mathrm{~m} / \mathrm{s}(1129 \mathrm{ft} / \mathrm{s})$, so you can figure the distance to the article utilizing this recipe:

\section{$0,5 *(344 * \mathrm{~m} / \mathrm{s} *[$ reflection time $])=$ distance}

The engine driver circuit, or H-Bridge, is utilized to control the speed and course of the DC engines. It has two gulfs that should be associated with a different DC power source (engines draw hefty current, and can't be provided straightforwardly from the regulator), two arrangements of yields for each engine (positive and negative), two empower pins for each set of yields, and two arrangements of pins for the bearing control of each engine outlet. This double bidirectional engine driver depends on the exceptionally well known L298 Dual H-Bridge Motor Driver IC. This module permits you to effectively and freely control two engines both way.

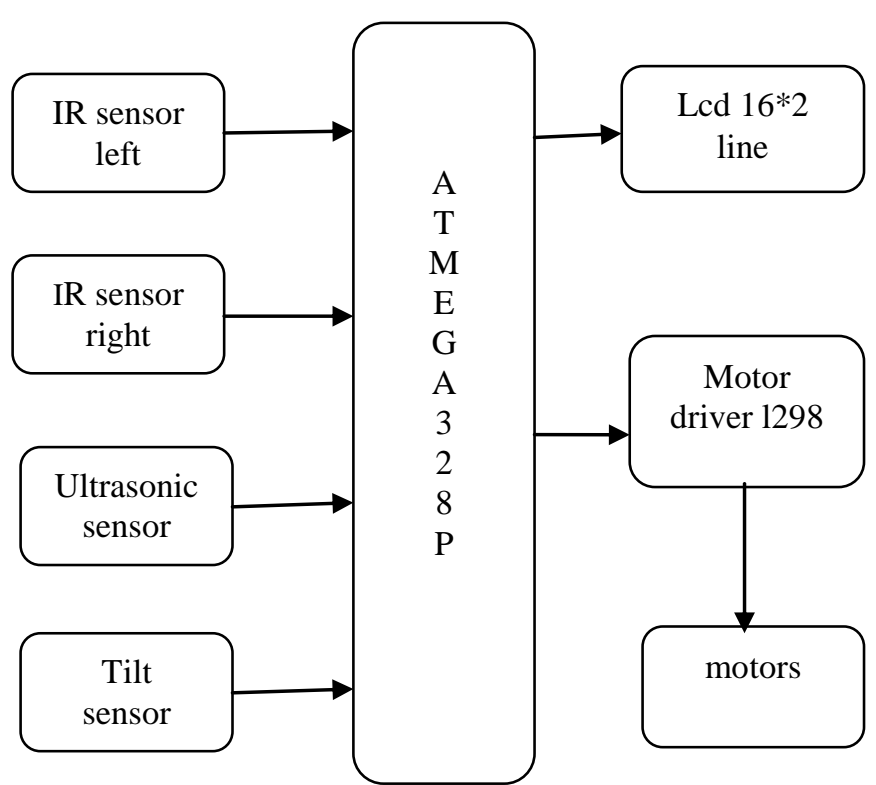

Fig. 2. The block diagram of path providing method.

The subsequent contention shows the time frame esteem. This number is utilized to decide the PWM time frame. When Timer 2 arrives at the number equivalent to the contention, the PWM yield changes from high to low. The accompanying condition gives the complete PWM period

$$
\text { PWM-period }=\frac{1}{\text { elock }} \times 4 \times \text { T2 DIV } \times(\text { period }+1)
$$

In order to discover the on time for a particular speed,

$$
\text { On-time }=\frac{\text { Dutycyclex PWMperiod }}{10036}
$$

The relation between the speed of a motor at no load and duty cycle is;

$$
\text { Duty-cycle }=\frac{S_{\text {peed }}+100}{2}
$$

Numerous elements overall prevent a robot from moving in straight way. They range from Mechanical shortcomings and distinctive reaction of engines to comparable contribution to the way that rubbing is non uniform in a genuine situation. Because of this, during the execution of the calculations as planned above was not clear rather couple of changes were made. The most remarkable ones are : 
- The PID regulator was altered to address for various reaction by the engines. For this as opposed to having the control exertion took care of to them, we had a changed exertion for each engine.

- We needed to utilize integral channel to put a beware of arbitrary mistaken qualities from the sensors.

- $90^{\circ}$ turns were additionally carried out.

- Reverse divider following was additionally carried out for which certain changes to the framework were made. In particular the expansion of another distance sensor.

- Many a mixes of the above calculations were additionally tried and can be proficiently carried out for various instances of utilization. As a rule the best calculations will be a half and half one instead of an unadulterated one.

\section{SPEED CONTROL}

\section{A. PID controller}

The Proportional Integral Derivative Controller (PID) has been generally utilized for most modern interaction, because of its effortlessness and adequacy in charge. This kind of regulator is regularly utilized in level, stream, temperature, and vehicular frameworks, just as electric engines. Moreover, the plan of the PID regulator is viewed as simple to execute, since it is simply important to tune three boundaries and tuning strategies can be performed naturally. $\mathrm{P}, \mathrm{I}$, and $\mathrm{D}$ represent relative, vital, and subsidiary. As a rule the relative $(\mathrm{P})$ part of the control calculation gives the vast majority of the "push" to get things going. The necessary (I) term is utilized to follow up on little mistakes and power continuous changes over the long run. The subsidiary (D) a piece of the condition acts to moist motions or sudden changes in the control signal. The D expression is typically attempts to go against unexpected changes brought about by the $\mathrm{P}$ expression. PID regulator is a conventional control circle criticism instrument (regulator) broadly utilized in mechanical control framework a PID is the most ordinarily utilized input regulator. Compute a blunder esteem as the distinction between estimated measure variable and an ideal reaction. The regulator endeavors to limit the mistake by changing the interaction control input. The PID regulator estimation (calculation) includes three consistent boundaries called the relative (P), fundamental (I) and subordinate (D) values, these qualities can be deciphered regarding time. $\mathrm{P}$ relies upon the current blunder, I on the aggregation of past mistake, and D is an expectation of future blunder, in light of current pace of progress. The weighted amount of these three activities is utilized to change the cycle by means of a control component like the situation of a control valve, or force provided to a warming component. PID regulator comprises of three terms, in particular relative, essential, and subordinate control. The joined activity of these three regulators gives a control system for measure control. PID regulator controls the cycle factors like pressing factor, speed, temperature, stream, and so on A portion of the applications use PID regulators in course networks where at least two PID's are utilized to accomplish control.
A PID control for electric vehicles subject to enter armature voltage and rakish speed signal imperatives is proposed. Relative vital subordinate control is an input regulator including three separate steady boundaries, the corresponding, the basic and subsidiary qualities, meant P, I, and D, in this way now and then called three-term control.The Simulink model of vehicle constrained by PID regulator is appeared in Fig. 3. By consolidating the benefits like snappier reaction time (because of P-just control), alongside the diminished/zero counterbalance (because of Ijust control)and forecast of unsettling influences to the framework (because of D-control) limits the mistake between some client characterized set point and the deliberate cycle variable. The impelling sign for the PID regulator and the exchange work.

The variable $\mathrm{e}(\mathrm{s})$ represent the mistake signal used to follow the reference signal. The variable e(s) is the contrast between the ideal reference input set point esteem $\mathrm{r}(\mathrm{s})$ and the real yield $\omega(\mathrm{s})$. This blunder signal is shipped off the PID regulator and afterward regulator assesses the subordinate and the vital of the mistake signal. The yield signal (u) of the regulator gets equivalent to the relative addition $(\mathrm{Kp})$ times the extent of the blunder in addition to the essential increase (Ki) times the fundamental of the mistake in addition to the subsidiary increase $(\mathrm{Kd})$ times the subordinate of the blunder. The sign (u) will currently be shipped off the plant, and get the new created yield $\omega(\mathrm{s})$. This yield will be again return back to the sensor to decide the new blunder signal $\mathrm{e}(\mathrm{s})$. The new mistake sign will be again shipped off the regulator and processes both subordinate and fundamental once more. The above cycle rehashes in a similar way. The sign (u) can be addressed as follow

$$
u=K p e+K i \int e d t+K d \frac{d l e}{d t}
$$

The transfer function of PID Controller

$$
G c=K P+\frac{K I}{S}+K_{D} S
$$

The algorithm works on the error produced from the difference between the reference speed and the actual speed. PID parameters Proportional gain Kp, Integral Gain Ki, and Derivative Gain Kd affects system's overall performance. For this reason selecting right parameters for a system is a difficult process and can be done by using several tuning methods which includes manual tuning, Ziegler-Nicholas tuning and Cohen-coon tuning. Normally a mathematical model of the system is designed along with PID controller and the system performance is observed with applied set of values of PID parameters to finalize the best suited values. Following are the effects of PID parameters (Kp, Ki, and $\mathrm{Kd})$

- System Rise time will be reduced by Kp, it provides faster response in variable load condition

- Steady state error will be reduced by $\mathrm{Ki}$, hence the motor speed is pushed near to reference speed

- Settling time and overshoot will be reduced by $\mathrm{Kd}$, hence provides faster response.

\section{B. Fuzzy Logic}

The principal original paper of "Fuzzy Logic" was given by Prof. Lotfi Zadeh in 1965 on Sets the Foundation of the Fuzzy Set hypothesis. Fuzzy frameworks are 
information/rule based frameworks taking after human dynamic with a capacity to create exact arrangements from certain or surmised data. While different methodologies require exact conditions to show true practices; fuzzy rationale can oblige the ambiguities of true human language and rationale by giving an instinctive technique to portraying frameworks in human terms and changing over it into successful models. Being non-direct in nature, it is more hard to set the additions in fuzzy rationale regulator (FLC), when contrasted with PID regulators In this paper, two sorts of arrangements are applied, fundamentally, fuzzy PD and fuzzy PD+I. The Mamdani based FLC has two-input , partitioned into seven states and one-yield (u). The control execution relies upon the received principle base, in this way, 7 information sources and 7 yield participation capacities are portrayed in 49 Fuzzy-in the event that rules given in Table II. Note that states are addressed as truncations like "NS" for "negative little, etc. as it assists with keeping the phonetic portrayals short yet exact.

- Fuzzification.

- Knowledge base.

- Fuzzy surmising.

\section{- De-Fuzzification}

During the Fuzzification stage the info plant boundaries are changed over into phonetic fuzzy variable. Second part Knowledge base otherwise called master information gives the vital data set to outline the phonetic control rules. The phonetic guideline base portrays the control objective and control strategy. This part gives phonetic control rules dependent on master information data set. The third part Inference Engine is the cerebrum of Fuzzy Logic Controller where the dynamic and legitimate thinking like human sort dynamic is recreated dependent on fuzzy insight, fuzzy deduction control rules and fuzzy ramifications. The fourth part De-Fuzzification plays out the undertaking of scale planning of the scope of estimations of yield factors into comparing universe of talk to yield a non fuzzy control activity dependent on construed fuzzy control rule. This change is performed utilizing fuzzy participation work. The quantity of participation work and their shapes are controlled by client at beginning stage as it were.

Fuzzification is technique for changing a fresh amount into a fuzzy amount. This can be accomplished by recognizing the different referred to fresh and deterministic amounts as totally nondeterministic and very unsure in nature. This vulnerability may have arisen as a result of ambiguity and imprecision which at that point lead the factors to be addressed by an enrollment work as they can be fuzzy in nature.

De-fuzzyfication is the reversal of fuzzification, there the planning is done to change over the fresh outcomes into fuzzy outcomes yet here the planning is done to change over the fuzzy outcome into fresh outcomes This cycle is equipped for producing a non-fuzzy control activity which delineates the chance conveyance of a gathered fuzzy control activity.

De-fuzzification interaction can likewise be treated as the adjusting cycle, where fuzzy set having a gathering of participation esteems on the unit stretch diminished to a solitary scalar amount.

\begin{tabular}{|l|l|l|l|l|l|l|l|}
\hline $\begin{array}{l}\text { E/C } \\
\text { E }\end{array}$ & DB & DH & DI & NI & AS & AT & AI \\
\hline DB & DB & DB & DB & DB & DH & DI & NI \\
\hline DH & DB & DB & DB & DH & DI & NI & AS \\
\hline DI & DB & DB & DH & DI & NI & AS & AT \\
\hline NI & DB & DH & DI & NI & AS & AT & AI \\
\hline AS & DH & DI & NI & AS & AT & AI & AI \\
\hline AT & DI & NI & AS & AT & AI & AI & AI \\
\hline AI & NI & AS & AT & AI & AI & AI & AI \\
\hline
\end{tabular}

Fig. 3. Rule base for controller

\section{CONCLUSION}

In this research paper we proposed way following regulator for a self-governing vehicle with four wheels, the regulator depends on fuzzy logic, can offer really following adaptability . After a few tried that we did we saw that the beginning stage and the underlying direction affected on the direction following, consequently we need to pick the underlying condition to diminish the estimation of the mistake, the proposed model can be likewise utilized in the platooning project later on works we plan, to coordinate the proposed strategy to accomplish a train of vehicles. The electric vehicle was demonstrated best comportment and strength during various street way. $m$. The proposed Fuzzy regulator model improve the driving wheels speeds control with high precision either in level streets or bended ones .The aggravations don't influence the exhibitions of the driving engines and the control law's productivity gives a decent dynamic attributes of the foothold chain. The significant advertisement vantages of a vehicle utilizing free wheel control are the capacity to cross steep slants, and the capacity to have bigger haggles load conveying and dissemination of burden contrasted with traditional vehicles of the kind.

\section{ACKNOWLWDGEMENT}

First of all we thanks to our Principal Prof. P.B. Ghewari, for giving the help. Special thanks to our Head of Department and our project guide, Prof. A.A. Malgave, for consistent support, motivation and guidance that helped us completing our project successfully.

\section{REFERENCES}

[1] Green Growth Management by Using Arm Con-troller, B Yogesh Ramdas et al Int. Journal of En-gineering Research and Applications ISSN : 2248-9622, Vol. 4, Issue 3( Version 1), March 2014, pp.360363.

[2] D.S.Suresh, Jyothi Prakash K V, Rajendra C J, ”Automated Soil Testing Device", ITSI Transac-tions on Electrical and Electronics Engineering (ITSI-TEEE) ISSN (PRINT): 2320 - 8945, Vol-ume - 1, Issue $-5,2013$.

[3] Soil Testing in India", Department of Agriculture \& Co-operation, Ministry of Agriculture, Govern-ment of India, New Delhi, January, 2011.

[4] Sneha J. Bansod, Shubhadha Thakre, "Near Infra-red Spectroscopy based Soil Nitrogen measure-ment", International Journal of Current Engineer-ing and Technology E-ISSN 2277 - 4106, P-ISSN 2347 5161.

[5] K. Prema, N. Senthil Kumar, and K.A. Sunitha, (2009), Online Temperature Control Based On Virtual Instrumentation, IEEE. 